\title{
New Vacuum Ultraviolet Wavelengths and Revised Energy Levels in the Second Spectrum of Zinc (ZnII)
}

\author{
William C. Martin and Victor Kaufman \\ Institute for Basic Standards, National Bureau of Standards, \\ Washington, D.C. 20234
}

(September 23, 1969)

\begin{abstract}
Spectra from sliding spark discharges between zinc electrodes and from a hollow-cathode source were photographed in the range 2105-1400 $\AA$ with the NBS $10.7 \mathrm{~m}$ vacuum spectrograph. New measurements of $130 \mathrm{Zn}$ II lines in this region, combined in some cases with previous measurements in the air region, were used to derive revised positions for the known levels. Wavelengths calculated from these levels are given for 267 lines in the region 2570-730 $\AA$. The line list also includes unclassified Zn II lines below $2570 \AA$, based on the most complete description of this spectrum [A. M. Crooker and K. A. Dick, Can. J. Phys. 46, 1241 (1968)]. A few additions to the line list of Crooker and Dick above $2700 \AA$ are given. The line and level lists take into account recent work the National Bureau of Standards on the Zn II analysis.
\end{abstract}

Key words: Atomic energy levels of $\mathrm{Zn}$ II; ionized zinc; spectral wavelengths of $\mathrm{Zn}$ II.

\section{Introduction}

An improved description of the Zn II spectrum has recently been given by Crooker and Dick $[1],{ }^{1}$ who also extended and revised the earlier analyses $[2,3]$. From a combination array made in connection with a theoretical fitting of some $\mathrm{Zn}$ II levels [4, 5], we decided that a resolution of several blends in the vacuum ultraviolet spectrum was needed. It appeared likely that the resolution available in this laboratory was sufficiently greater than that obtained by Crooker and Dick to make worthwhile a reobservation of $\mathrm{Zn} \mathrm{II}$ in the indicated region. The resulting new wavelengths are reported here, together with a list of revised energy levels partially based on them.

\section{Observations}

The spectra were photographed with the NBS $10.7 \mathrm{~m}$ Eagle-mounting vacuum spectrograph. A 1200 grooves $/ \mathrm{mm}$ grating used in the first order gave a plate factor of $0.78 \AA / \mathrm{mm}$. One source was a sliding spark between zinc electrodes, in series with an auxiliary rotating spark gap and appropriate inductance,

${ }^{1}$ Figures in brackets indicate the literature references at the end of this paper. resistance, and capacitance charged to $500 \mathrm{~V}[6]$. Three different exposures were made: ${ }^{2}$

$$
\begin{array}{ll}
S_{1}, \text { hot spark } & 2105-1714 \AA \\
S_{2}, \text { mild spark } & 2105-1714 \AA \\
S_{3}, \text { mild spark } & 2105-1400 \AA .
\end{array}
$$

The hot spark gave the stronger lines of Zn IV and Zn III and also gave our most complete Zn II spectrum. The mild sparks showed no Zn IV lines, and were strong sources of $\mathrm{ZnI}$ and of $\mathrm{Zn}$ II lines having low or medium excitation energies. Spectra for standard wavelengths (CI, NI, and GeI[7]) were photographed only on the $S_{3}$ spectrograms. Zinc wavelengths obtained from this exposure were the principal standards for reducing the other two exposures.

We also obtained measurements of spectra from a water-cooled aluminum hollow-cathode source that contained zinc (as an impurity in the aluminum) and copper, silicon, and germanium (for standard wavelengths [7]). The discharge current was $0.8 \mathrm{~A}$ through a carrier gas of helium at about 2 torr. Each of five different exposures ( 3 to $30 \mathrm{~min}$ ) covered the range $2105-1400 \AA$.

\footnotetext{
${ }^{2}$ Typical circuit parameters for hot and mild sparks are given in [6]. We used peak currents varying from several hundred amperes $\left(S_{1}\right)$ to a few amperes $\left(S_{2}\right)$.
} 


\section{Line List and Level Values}

Table 1 contains more than $300 \mathrm{Zn}$ II lines in the range 2570-730 $\AA$. Crooker and Dick [1] have obtained by far the most complete spectrum in this region, and the observed wavelengths for about two-thirds of these lines are taken from their list. The observed wavelengths for 130 lines are from our measurements (2105-1400 $\AA$, with a number $1-4$ in the first column). We derived most of the level values in table 2 using these new measurements combined with a few previous measurements at longer wavelengths where this appeared consistent or was necessary. The calculated wavelengths given for the 267 classified lines in table 1 were obtained from the revised levels. They are recommended as the most accurate wavelengths now available for $\mathrm{Zn}$ II in this region. An " $\mathrm{X}$ " in the calculatedwavelength column indicates that the corresponding level separation was based entirely on the observed wavelength for this transition.

The resulting wavelengths are categorized as follows (numbers correspond to those in the first column of Table 1):

1. The observed wavelength is from the hollow-cathode source. Most of these lines were measured in each of two to five different exposures. The estimated uncertainty of the calculated wavelength is $\pm 0.002 \AA$. (The different measurements for a single line usually agreed to within $\pm 0.001 \AA$, but this precision was not maintained in the accuracy as indicated by the Ritz principle.)

2. All the above applies except that the observed wavelength is based on a single measurement of a weak line. The observed wavelength still usually agrees with the (preferred) calculated value to within $0.003 \AA$.

3 . This indicates a line of the (2) type except that the corresponding energy level difference is based on this one measurement. The wavelength is probably accurate to $\pm 0.003 \AA$.

4. The observed wavelength is from our sliding spark sources. The Ritz-principle consistency of these lines is considerably poorer than for the hollow-cathode wavelength (see below). The observed and calculated wavelengths for unblended lines are different by about $0.005 \AA$ on the average, and by more than $0.015 \AA$ for a few lines. Most of the calculated wavelengths should be in error by less than $0.01 \AA$.

No number in the first column. The observed wavelength is from [1] or [9]. The calculated wavelengths for most of these lines are based on level differences derived from our sliding-spark measurements; the corresponding probable error is less than $0.01 \AA$.

The rms deviation of the standard wavelengths (from smooth reduction curves) for the spark spectrograms was about $0.002 \AA$. The much larger error for the resulting zinc measurements (type 4 lines, above) is not too surprising, since we had only one independent measurement of the spark spectrum against well-known reference wavelengths. The accuracy for some $\mathrm{Zn}$ II lines was also reduced by their width or diffuseness. More accurate wavelength measurements for some $\mathrm{Zn}$ II lines from a spark source would probably not be justified, because of sensitivity to Stark shifts. The $6 f^{2} \mathrm{~F}^{\circ}$ levels, for example, are depressed by about $1 \mathrm{~cm}^{-1}$ in the sliding-spark discharge. This accounts for most of the disagreement between the calculated and observed wavelengths for the line at 1430.27 $\AA$.

Except for the even levels above the limit, most of the level values in table 2 are consistent with the best observations to $\pm 0.2 \mathrm{~cm}^{-1}$. Values are given to two decimal places for some levels that appeared consistent to within $\pm 0.1 \mathrm{~cm}^{-1}$. Most of the levels above the limit are probably in error by less than $0.5 \mathrm{~cm}^{-1}$.

The revised levels give improved agreement between the calculated and the more accurately measured wavelengths over the entire spectrum. Disagreements between calculated and observed wavenumbers are much reduced (from $\sim 2 \mathrm{~cm}^{-1}$ in [1]) for several accurately measured air-region lines connecting $3 d^{10} f$ and $3 d^{10} d$ levels. The new wavelengths in the region 2000-2103 $\AA$, which contains the basic $3 d^{10} 4 s-3 d^{10} 4 p$ transitions and also the $3 d^{10} 4 p-3 d^{10} 4 d$ multiplet, are systematically longer than the values in [1] by more than $0.03 \AA$. Thus the revised values for the $4 p^{2} \mathrm{P}^{\circ}$ and $4 d^{2} \mathrm{D}$ levels are less than the values in [1] by about $0.7 \mathrm{~cm}^{-1}$ and $1.7 \mathrm{~cm}^{-1}$, respectively. This accounts for a systematic differencè (between the two lists) for all excited levels except those of the $3 d^{10} f$ and $3 d^{10} g$ configurations. The systematic difference that exists between our levels and the values of Shenstone and Gibson $[2,3]$ is smaller and in the opposite direction.

Estimated relative intensities ${ }^{3}$ and line characteristics from several different sources are given in table 1 , since they often help confirm the analysis. Comparing intensities of $\mathrm{Zn}$ II lines from the hot spark $\left(S_{1}\right)$ and the mild sparks $\left(S_{2}, S_{3}\right)$, we find in $S_{1}$ an enhancement of lines from levels above the ionization limit. Some of the weaker lines that appeared only in $S_{1}$ were clearly broadened relative to other lines, and the classifications indicate that this width arises from autoionization. We note especially that transitions from the four $3 d^{9} 4 s\left({ }^{3} \mathrm{D}\right) 5 s$ levels having $J=1 \frac{1}{2}$ or $2 \frac{1}{2}$ are always wide, analogous to the Cu I spectrum [8]. These same autoionizing transitions are quite strong in Dick's source $E_{1}$ and very weak or not observed in $E_{2} .^{4}$

An example of the usefulness of these excitation characteristics is the classification of the line at $1833.573 \AA$. We began this work mainly to attempt a resolution of this $4 p^{\prime}{ }^{4} \mathrm{~F}_{41 / 2}^{\circ}-5 s^{\prime}{ }^{4} \mathrm{D}_{31 / 2}$ transition from the $4 s^{2}{ }^{2} \mathrm{D}_{21 / 2}-4 f^{2} \mathrm{~F}^{\circ}$ line. The relative intensities of $1833.573 \AA$ in $S_{1}$ and $S_{2}, S_{3}$ (as compared, for example, with the now resolved transition from $4 f^{2} \mathrm{~F}^{\circ}$ ) show that it arises from a level above the limit. However, the level is not observably autoionization-broadened. These characteristics are consistent with a transition

\footnotetext{
${ }^{3}$ The relative intensities of 42 lines observed in the hollow-cathode spectrum were similar to those in the mildest spark $\left(S_{2}\right)$ spectrum, and are not given in table 1.

${ }^{4}$ In the region above $2385 \AA, E_{1}$ and $E_{2}$ in the table are Dick's [9] intensities $I_{4}$ and $I_{5}$, respectively; below $2385 \AA, E_{1}$ and $E_{2}$ are his intensities $I_{1}$ and $I_{2}$, respectively. Our staterespectively; below $2385 \mathrm{~A}, E_{1}$ and $E_{2}$ are his intensities $I_{1}$ and $I_{2}$, respectively. Our state-
ments about relative intensities refer to $I_{1}$ and $I_{2}$. Three autoionizing lines with apparently comparable intensities in these two sources are probably blends (see notes to table 1).
} 
TABLE 1. Zn II spectrum below $2570 \AA$

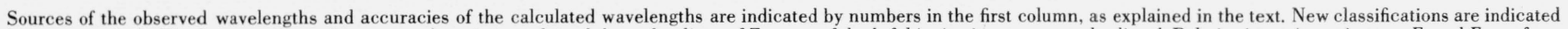

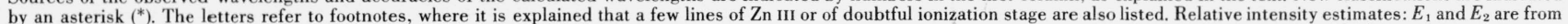

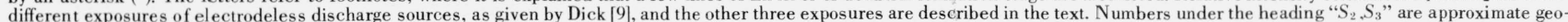

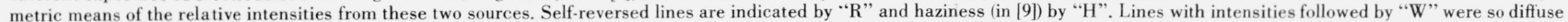
(on our plates) as to suggest autoionization broadening.

\begin{tabular}{|c|c|c|c|c|c|c|c|c|}
\hline \multirow{2}{*}{ Notes } & \multicolumn{2}{|c|}{$\lambda($ air),$\AA$} & \multicolumn{4}{|c|}{ Intensity } & \multirow{2}{*}{$\begin{array}{c}\sigma, \mathrm{cm}^{-1} \\
\text { obs. }\end{array}$} & \multirow{2}{*}{ Classification } \\
\hline & obs. & calc. & $E_{1}$ & $E_{2}$ & & & & \\
\hline * & $\begin{array}{l}2567.975 \\
2567.795 \\
2564.456 \\
2557.947 \\
2545.0\end{array}$ & $\begin{array}{l}.976 \\
.798 \\
.461 \\
.948 \\
.00\end{array}$ & $\begin{array}{r}50 \\
50 \\
30 \\
1000 \\
5\end{array}$ & $\begin{array}{r}30 \\
10 \\
30 \\
1000\end{array}$ & & & $\begin{array}{l}38929.5 \\
38932.3 \\
38982.9 \\
39082.13 \\
39281 .\end{array}$ & $\begin{array}{l}4 d^{2} \mathrm{D}_{21 / 2}-7 f^{2} \mathrm{~F}_{31 / 2}^{\circ} \\
4 d^{2} \mathrm{D}_{21 / 2}-7 f^{2} \mathrm{~F}_{21 / 2}^{\circ} \\
4 d^{2} \mathrm{D}_{11 / 2}-7 f^{2} \mathrm{~F}_{21 / 2}^{\circ} \\
4 p^{2} \mathrm{P}_{11 / 2}^{\circ}-5 s^{2} \mathrm{~S}_{1 / 2} \\
7 p^{2} \mathrm{P}_{11 / 2}^{\circ}-5 s^{\prime \prime}{ }^{\circ} \mathrm{D}_{21 / 2}\end{array}$ \\
\hline$b^{*}$ & $\begin{array}{l}2527.96 \\
2506.69 \\
2501.990 \\
2439.5 \\
2435.76\end{array}$ & $\begin{array}{l}8.01 ? \\
.70 \\
.990 \\
.53 \\
.79\end{array}$ & $\begin{array}{r}50 \\
10 \\
1000 \\
15 \\
15\end{array}$ & $\begin{array}{r}15 \\
5 \\
1000 \\
10\end{array}$ & & & $\begin{array}{l}39545.8 \\
39881.2 \\
39956.14 \\
40979.6 \\
41042.5\end{array}$ & $\begin{array}{c}4 d^{2} \mathrm{D}_{21 / 2}-9 p^{2} \mathrm{P}_{11 / 2}^{\circ} ? \\
4 s^{2}{ }^{2} \mathrm{D}_{11 / 2}-4 p^{\prime}{ }^{4} \mathrm{P}^{\circ}{ }_{11 / 2}^{\circ} \\
4 p^{2} \mathrm{P}_{1 / 2}^{\circ}-5 s^{2} \mathrm{~S}_{1 / 2}^{\circ} \\
4 s^{2}{ }^{2} \mathrm{D}_{21 / 2}-4 p^{\prime}{ }^{4} \mathrm{P}_{21 / 2}^{\circ} \\
4 d^{2} \mathrm{D}_{21 / 2}-8 f^{2} \mathrm{~F}^{\circ}\end{array}$ \\
\hline & $\begin{array}{l}2433.131 \\
2432.74 \\
2390.078 \\
2383.923 \\
2346.685\end{array}$ & $\begin{array}{l}.11 \\
.73 \\
\times \\
.949 \\
.68\end{array}$ & $\begin{array}{r}5 \\
10 \\
20 \\
30 \\
20\end{array}$ & $\begin{array}{r}1 \\
5 \\
30 \\
15 \\
12\end{array}$ & & & $\begin{array}{l}41086.8 \\
41093.4 \\
41826.9 \\
41934.9 \\
42600.3\end{array}$ & $\begin{array}{c}4 s^{2}{ }^{2} \mathrm{D}_{11 / 2}-4 p^{\prime}{ }^{4} \mathrm{P}_{1 / 2}^{\circ} \\
4 d^{2} \mathrm{D}_{11 / 2}-8 f^{2} \mathrm{~F}_{21 / 2}^{\circ} \\
4 s^{2}{ }^{2} \mathrm{D}_{11 / 2}-4 p^{\prime}{ }^{4} \mathrm{~F}_{21 / 2}^{\circ} \\
5 s^{2} \mathrm{~S}_{1 / 2}-4 p^{\prime \prime}{ }^{2} \mathrm{P}_{11 / 2}^{\circ} \\
4 s^{2}{ }^{2} \mathrm{D}_{21 / 2}-4 p^{\prime}{ }^{4} \mathrm{P}_{11 / 2}^{\circ}\end{array}$ \\
\hline $\begin{array}{l}* \\
*\end{array}$ & $\begin{array}{l}2336.493 \\
2273.150 \\
2212.402\end{array}$ & $\begin{array}{l}.48 \\
.175 \\
.437\end{array}$ & $\begin{array}{r}2 \\
50 \\
3\end{array}$ & $\begin{array}{c}0 \mathrm{H} \\
10 \\
1\end{array}$ & & & $\begin{array}{l}42786.1 \\
43978.2 \\
45185.7\end{array}$ & $\begin{array}{c}4 s^{2}{ }^{2} \mathrm{D}_{11 / 2}-4 p^{\prime}{ }^{4} \mathrm{~F}_{11 / 2}^{\circ} \\
5 s^{2} \mathrm{~S}_{1 / 2}-8 p{ }^{2} \mathrm{P}_{1 / 2}^{\circ} \\
5 s^{2} \mathrm{~S}_{1 / 2}-8 p{ }^{2} \mathrm{P}_{11 / 2}^{\circ}\end{array}$ \\
\hline & $\begin{array}{l}2210.176 \\
2203.511\end{array}$ & $\begin{array}{l}.201 \\
.453\end{array}$ & $\begin{array}{l}60 \\
15\end{array}$ & $\begin{array}{r}15 \\
5\end{array}$ & & & $\begin{array}{l}45231.2 \\
45368.0\end{array}$ & $\begin{array}{c}4 s^{2}{ }^{2} \mathrm{D}_{11 / 2}-4 p^{\prime}{ }^{2} \mathrm{~F}_{21 / 2}^{\circ} \\
5 s^{2} \mathrm{~S}_{1 / 2}-4 p^{\prime \prime}{ }^{2} \mathrm{P}_{1 / 2}^{\circ}\end{array}$ \\
\hline b & $\begin{array}{l}2163.475 \\
2159.060 \\
2150.539 \\
2147.419 \\
2122.741\end{array}$ & $\begin{array}{l}.511 \\
.085 \\
.58 \\
.43 \\
.1 / 5\end{array}$ & $\begin{array}{r}6 \\
2 \\
10 \\
75 \\
75\end{array}$ & $\begin{array}{l}8 \\
2 \\
6 \\
20 \mathrm{R} \\
12\end{array}$ & & & $\begin{array}{l}46207.4 \\
46301.9 \\
46485.3 \\
46552.9 \\
47094.0\end{array}$ & $\begin{array}{r}5 s^{2} \mathrm{~S}_{1 / 2}-4 p^{\prime \prime}{ }^{2} \mathrm{D}_{11 / 2}^{\circ} \\
4 s^{2}{ }^{2} \mathrm{D}_{11 / 2}-4 p^{\prime}{ }^{4} \mathrm{D}_{21 / 2}^{\circ} \\
4 p^{\prime}{ }^{2} \mathrm{D}_{21 / 2}^{\circ}-5 s^{\prime}{ }^{4} \mathrm{D}_{31 / 2} \\
4 s^{2}{ }^{2} \mathrm{D}_{11 / 2}-4 p^{\prime}{ }^{4} \mathrm{D}_{11 / 2}^{\circ} \\
4 s^{2}{ }^{2} \mathrm{D}_{11 / 2}-4 p^{\prime}{ }^{4} \mathrm{D}_{1 / 2}^{\circ}\end{array}$ \\
\hline $\mathrm{c}$ & 2116.318 & .35 & 5 & 10 & & & 47236.9 & $4 p^{\prime}{ }^{2} \mathrm{D}_{21 / 2}^{\circ}-5 s^{\prime}{ }^{4} \mathrm{D}_{21 / 2}$ \\
\hline \multicolumn{3}{|c|}{$\lambda(\mathrm{vac}), \AA$} & \multicolumn{4}{|c|}{ Intensity } & & \\
\hline & obs. & calc. & $E_{1}$ & $E_{2}$ & $S_{1}$ & $S_{2}, S_{3}$ & & \\
\hline $\begin{array}{l}1 \\
1 \\
3 \\
4\end{array}$ & $\begin{array}{l}2102.844 \\
2100.604 \\
2085.511 \\
2082.191 \\
2081.115\end{array}$ & $\begin{array}{l}.842 \\
\times \\
\times \\
.246 \\
.111\end{array}$ & $\begin{array}{r}200 \\
300 \\
30 \\
4 \\
25\end{array}$ & $\begin{array}{c}200 \mathrm{R} \\
300 \mathrm{R} \\
15 \\
18\end{array}$ & $\begin{array}{r}400 \\
700 \\
2 \\
3\end{array}$ & $\begin{array}{r}400 \\
700 \\
2 \\
3\end{array}$ & $\begin{array}{l}47554.65 \\
47605.36 \\
47949.88 \\
48026.3 \\
48051.2\end{array}$ & $\begin{array}{c}4 p^{2} \mathrm{P}_{11 / 2}^{\circ}-4 d^{2} \mathrm{D}_{11 / 2} \\
4 p^{2} \mathrm{P}_{11 / 2}^{\circ}-4 d^{2} \mathrm{D}_{21 / 2} \\
4 s^{2}{ }^{2} \mathrm{D}_{21 / 2}-4 p^{\prime}{ }^{2} \mathrm{~F}_{21 / 2}^{\circ} \\
4 p^{\prime}{ }^{2} \mathrm{D}_{11 / 2}^{\circ}-5 s^{\prime}{ }^{4} \mathrm{D}_{21 / 2} \\
4 s^{2}{ }^{2} \mathrm{D}_{11 / 2}-4 p^{\prime}{ }^{2} \mathrm{P}_{1 / 2}^{\circ}\end{array}$ \\
\hline $\begin{array}{l}4 \mathrm{~b} \\
3 \\
1 \\
1 \\
1\end{array}$ & $\begin{array}{l}2080.596 \\
2077.070 \\
2064.887 \\
2062.662 \\
2057.471\end{array}$ & $\begin{array}{l}.600 \\
\times \\
.890 \\
.664 \\
.470\end{array}$ & $\begin{array}{r}50 \\
20 \\
200 \\
300\end{array}$ & $\begin{array}{c}20 \\
15 \\
300 \mathrm{R} \\
500 \mathrm{R} \\
25 \mathrm{R}\end{array}$ & $\begin{array}{c}8 \mathrm{~W} \\
600 \\
1200 \mathrm{R} \\
80\end{array}$ & $\begin{array}{c}1 \\
600 \\
1200 \mathrm{R} \\
80\end{array}$ & $\begin{array}{l}48063.2 \\
48144.74 \\
48428.80 \\
48481.04 \\
48603.36\end{array}$ & $\begin{array}{c}4 p^{\prime}{ }^{2} \mathrm{D}_{21 / 2}^{\circ}-5 s^{\prime}{ }^{4} \mathrm{D}_{11 / 2} \\
4 s^{2}{ }^{2} \mathrm{D}_{21 / 2}-4 p^{\prime}{ }^{4} \mathrm{D}_{31 / 2}^{\circ} \\
4 p{ }^{2} \mathrm{P}_{1 / 2}^{\circ}-4 d^{2} \mathrm{D}_{11 / 2} \\
4 s^{2} \mathrm{~S}_{1 / 2}-4 p^{2} \mathrm{P}_{1 / 2}^{\circ} \\
4 s^{2}{ }^{2} \mathrm{D}_{11 / 2}-4 p^{\prime}{ }^{2} \mathrm{D}_{11 / 2}^{\circ}\end{array}$ \\
\hline 1 & $\begin{array}{l}2045.594 \\
2044.588 \\
2039.962 \\
2037.479 \\
2029.543\end{array}$ & $\begin{array}{l}.626 \\
\times \\
.556\end{array}$ & $\begin{array}{r}5 \\
30 \\
60 \\
8 \\
35\end{array}$ & $\begin{array}{c}3 \\
12 \\
25 \mathrm{R} \\
8 \\
12\end{array}$ & 50 & 50 & $\begin{array}{l}48885.6 \\
48909.6 \\
49020.52 \\
49080.3 \\
49272.2\end{array}$ & $\begin{array}{l}4 p^{\prime}{ }^{2} \mathrm{~F}_{31 / 2}^{\circ}-5 s^{\prime}{ }^{4} \mathrm{D}_{31 / 2} \\
4 s^{2}{ }^{2} \mathrm{D}_{21 / 2}-4 p^{\prime}{ }^{4} \mathrm{D}_{21 / 2}^{\circ} \\
4 s^{2}{ }^{2} \mathrm{D}_{21 / 2}-4 p^{\prime}{ }^{4} \mathrm{D}_{11 / 2}^{\circ}\end{array}$ \\
\hline $\begin{array}{l}1 \\
4 b\end{array}$ & $\begin{array}{l}2026.137 \\
2017.099 \\
2013.616\end{array}$ & $\begin{array}{l}.136 \\
.129 \\
.677\end{array}$ & $\begin{array}{r}300 \\
25 \\
15\end{array}$ & $\begin{array}{l}500 \mathrm{R} \\
15 \\
15\end{array}$ & $\begin{array}{r}1500 \mathrm{R} \\
2 \mathrm{~W}\end{array}$ & $1500 \mathrm{R}$ & $\begin{array}{l}49355.00 \\
49576.1 \\
49661.9\end{array}$ & $\begin{array}{r}4 s^{2} \mathrm{~S}_{1 / 2}-4 p{ }^{2} \mathrm{P}_{11 / 2}^{\circ} \\
4 p^{\prime}{ }^{4} \mathrm{D}_{21 / 2}^{\circ}-5 s^{\prime}{ }^{4} \mathrm{D}_{31 / 2} \\
4 p^{\prime}{ }^{2} \mathrm{~F}_{31 / 2}^{\circ}-5 s^{\prime}{ }^{4} \mathrm{D}_{21 / 2}^{\circ}\end{array}$ \\
\hline
\end{tabular}


TABLE 1. Zn II spectrum below $2570 \stackrel{A-C o n t i n u e d}{ }$




TABLE 1. Zn II spectrum below $2570 \AA$ - Continued

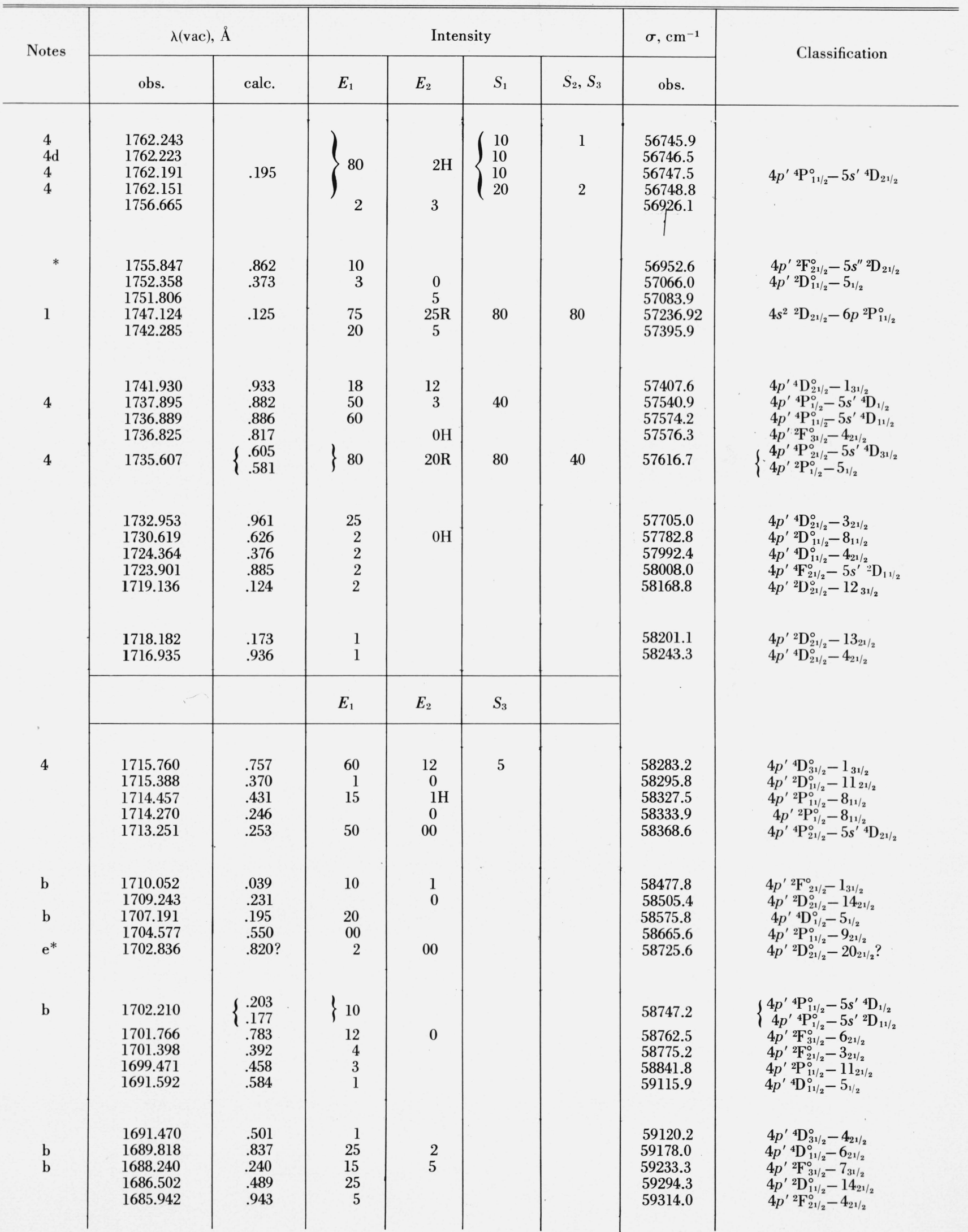




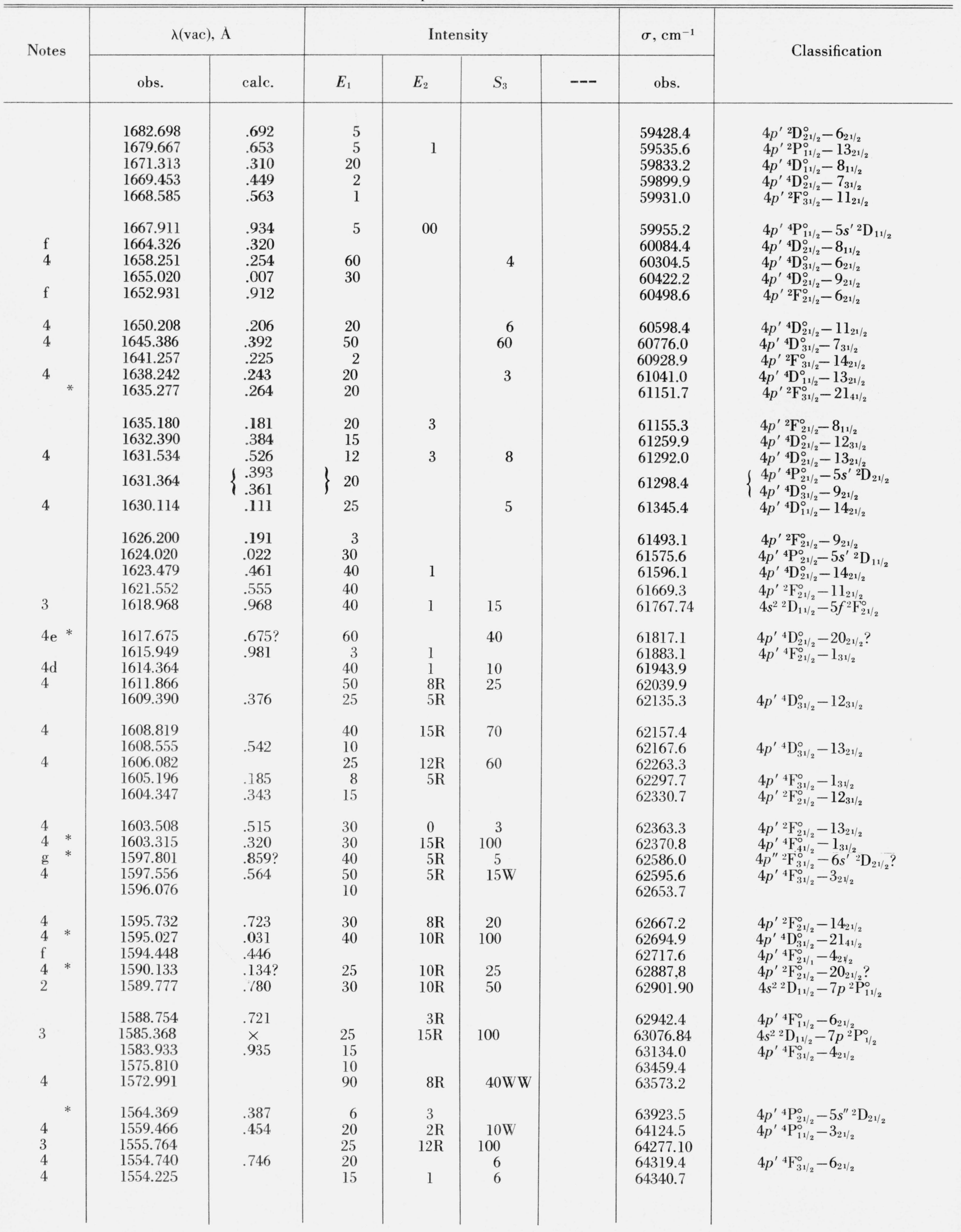


TABLE 1. Zn II spectrum below $2570 \AA$-Continued

\begin{tabular}{|c|c|c|c|c|c|c|c|c|c|}
\hline \multirow{2}{*}{\multicolumn{2}{|c|}{ Notes }} & \multicolumn{2}{|c|}{$\lambda(\mathrm{vac}), \AA$} & \multicolumn{4}{|c|}{ Intensity } & \multirow{2}{*}{$\begin{array}{c}\sigma, \mathrm{cm}^{-1} \\
\text { obs. }\end{array}$} & \multirow{2}{*}{ Classification } \\
\hline & & obs. & calc. & $E_{1}$ & $E_{2}$ & $S_{3}$ & --- & & \\
\hline $\begin{array}{l}3 \\
4 \\
4 \\
4 \\
4\end{array}$ & & $\begin{array}{l}1550.936 \\
1548.957 \\
1548.434 \\
1547.800 \\
1546.650\end{array}$ & $\begin{array}{l}.937 \\
.971 \\
.426\end{array}$ & $\begin{array}{l}25 \\
15 \\
20 \\
20 \\
25\end{array}$ & $\begin{array}{r}10 \mathrm{R} \\
\\
5 \mathrm{R} \\
5 \mathrm{R} \\
12 \mathrm{R}\end{array}$ & $\begin{array}{l}80 \\
3 \mathrm{~W} \\
20 \\
20 \\
100\end{array}$ & & $\begin{array}{l}64477.19 \\
64559.6 \\
64581.4 \\
64607.8 \\
64655.9\end{array}$ & $\begin{array}{l}4 s^{2}{ }^{2} \mathrm{D}_{21 / 2}-5 f^{2} \mathrm{~F}_{31 / 2}^{\circ} \\
4 p^{\prime}{ }^{4} \mathrm{~F}_{21 / 2}-8_{11 / 2}^{\circ} \\
4 p^{\prime}{ }^{4} \mathrm{P}_{1 / 2}^{\circ}-5_{1 / 2}\end{array}$ \\
\hline $\begin{array}{l}4 \\
4 \\
4 \\
4 \\
4\end{array}$ & & $\begin{array}{l}1546.460 \\
1545.087 \\
1544.922 \\
1543.428 \\
1543.037\end{array}$ & $\begin{array}{l}.465 \\
\\
.434 \\
.034\end{array}$ & $\begin{array}{l}20 \\
20 \\
25 \\
25 \\
25\end{array}$ & $\begin{array}{r}10 \mathrm{R} \\
8 \mathrm{R} \\
15 \mathrm{R} \\
10 \mathrm{R} \\
10 \mathrm{R}\end{array}$ & $\begin{array}{r}10 \\
2 \\
50 \\
40 \\
50\end{array}$ & & $\begin{array}{l}64663.8 \\
64721.3 \\
64728.2 \\
64790.8 \\
64807.3\end{array}$ & $\begin{array}{c}4 p^{\prime}{ }^{4} \mathrm{P}_{11 / 2}^{\circ}-4{ }_{21 / 2} \\
4 p^{\prime}{ }^{4} \mathrm{~F}_{31 / 2}^{\circ}-7_{31 / 2} \\
4 p^{\prime}{ }^{4} \mathrm{~F}_{11 / 2}^{\circ}-13_{21 / 2}\end{array}$ \\
\hline $\begin{array}{ll}4 & \\
4 & * \\
4 & \\
2 & \\
4 & \end{array}$ & * & $\begin{array}{l}1542.531 \\
1541.707 \\
1540.895 \\
1540.120 \\
1536.726\end{array}$ & $\begin{array}{l}.709 \\
.901 \\
.122 \\
.738\end{array}$ & $\begin{array}{l}20 \\
30 \\
35 \\
30 \\
25\end{array}$ & $\begin{array}{l}10 \mathrm{R} \\
12 \mathrm{R} \\
15 \mathrm{R} \\
10 \mathrm{R} \\
10 \mathrm{R}\end{array}$ & $\begin{array}{l}2 \mathrm{WW} \\
40 \\
80 \\
50 \\
20\end{array}$ & & $\begin{array}{l}64828.5 \\
64863.2 \\
64897.3 \\
64930.01 \\
65073.4\end{array}$ & $\begin{array}{l}4 p^{\prime}{ }^{4} \mathrm{~F}_{41 / 2}^{\circ}-7_{31 / 2} \\
4 p^{\prime}{ }^{4} \mathrm{~F}_{21 / 2}^{\circ}-9_{21 / 2}^{\circ} \\
4 s^{2}{ }^{2} \mathrm{D}_{11 / 2}-4 p^{\prime \prime 2} \mathrm{P}_{11 / 2}^{\circ} \\
4 p^{\prime}{ }^{4} \mathrm{~F}_{21 / 2}^{\circ}-11_{21 / 2}^{\circ}\end{array}$ \\
\hline $\begin{array}{l}4 \\
1 \\
4 \\
4\end{array}$ & * & $\begin{array}{l}1535.823 \\
1535.081 \\
1531.394 \\
1531.088 \\
1530.620\end{array}$ & $\begin{array}{l}.818 \\
.085 \\
.422 \\
.082 \\
.639 ?\end{array}$ & $\begin{array}{l}30 \\
40 \\
25 \\
30 \\
25\end{array}$ & $\begin{array}{r}15 \mathrm{R} \\
40 \mathrm{R} \\
8 \mathrm{R} \\
8 \mathrm{R}\end{array}$ & $\begin{array}{c}30 \\
200 \\
3 \mathrm{~W} \\
30\end{array}$ & & $\begin{array}{l}65111.7 \\
65143.14 \\
65300.0 \\
65313.0 \\
65333.0\end{array}$ & $\begin{array}{l}4 p^{\prime}{ }^{4} \mathrm{~F}_{11 / 2}^{\circ}-14_{21 / 2} \\
4 p^{2} \mathrm{P}_{11 / 2}-6 s^{2} S_{1 / 2} \\
4 p^{\prime}{ }^{4} \mathrm{P}_{1 / 2}^{\circ}-8_{11 / 2} \\
4 p^{\prime}{ }^{4} \mathrm{~F}_{31 / 2}^{\circ}-9_{21 / 2} \\
4 p^{\prime}{ }^{4} \mathrm{~F}_{11 / 2}^{\circ}-20_{21 / 2}^{\circ} ?\end{array}$ \\
\hline $\begin{array}{l}4 \\
4 \\
4\end{array}$ & & $\begin{array}{l}1528.759 \\
1528.550 \\
1527.915 \\
1527.236 \\
1527.125\end{array}$ & .910 & $\begin{array}{l}25 \\
30 \\
30 \\
20 \\
25\end{array}$ & $\begin{array}{l}5 \mathrm{R} \\
10 \mathrm{R} \\
15 \mathrm{R} \\
10 \mathrm{R} \\
10 \mathrm{R}\end{array}$ & $\begin{array}{r}9 \\
40 \\
100\end{array}$ & & $\begin{array}{l}65412.5 \\
65421.5 \\
65448.7 \\
65477.8 \\
65482.5\end{array}$ & $4 p^{\prime}{ }^{4} \mathrm{P}_{21 / 2}^{\circ}-1_{31 / 2}$ \\
\hline $\begin{array}{l}4 \\
1 \\
4 \\
4 \\
4\end{array}$ & & $\begin{array}{l}1526.969 \\
1523.903 \\
1521.276 \\
1520.998 \\
1520.527\end{array}$ & $\begin{array}{r}.972 \\
.903 \\
.271 \\
1.003 \\
.526\end{array}$ & $\begin{array}{l}30 \\
40 \\
50 \\
40 \\
40\end{array}$ & $\begin{array}{r}10 \mathrm{R} \\
30 \mathrm{R} \\
8 \mathrm{R} \\
8 \mathrm{R} \\
10 \mathrm{R}\end{array}$ & $\begin{array}{r}15 \\
150 \\
40 \\
15 \\
50\end{array}$ & & $\begin{array}{l}65489.2 \\
65620.97 \\
65734.3 \\
65746.3 \\
65766.7\end{array}$ & $\begin{array}{l}4 p^{\prime}{ }^{4} \mathrm{~F}_{31 / 2}^{\circ}-11_{21 / 2} \\
4 s^{2}{ }^{2} \mathrm{D}_{21 / 2}-7 p^{2} \mathrm{P}_{11 / 2}^{\circ} \\
4 p^{\prime}{ }^{4} \mathrm{~F}_{21 / 2}^{\circ}-12_{31 / 2} \\
4 p^{\prime}{ }^{4} \mathrm{P}_{21 / 2}^{\circ}-3_{21 / 2}^{\circ} \\
4 p^{\prime}{ }^{4} \mathrm{~F}_{21 / 2}^{\circ}-13_{21 / 2}^{\circ}\end{array}$ \\
\hline $\begin{array}{l}4 \\
1 \\
4 \\
4\end{array}$ & & $\begin{array}{l}1520.022 \\
1518.631 \\
1514.763 \\
1514.479 \\
1513.522\end{array}$ & $\begin{array}{l}.039 \\
.628 \\
.761 \\
.519\end{array}$ & $\begin{array}{l}25 \\
30 \\
50 \\
30 \\
20\end{array}$ & $\begin{array}{r}15 \mathrm{R} \\
8 \mathrm{R} \\
8 \mathrm{R}\end{array}$ & $\begin{array}{r}3 \\
120 \\
25 \\
20\end{array}$ & & $\begin{array}{l}65788.5 \\
65848.8 \\
66016.93 \\
66029.3 \\
66071.1\end{array}$ & $\begin{array}{l}4 p^{\prime}{ }^{4} \mathrm{P}_{11 / 2}^{\circ}-5_{1 / 2} \\
4 p^{\prime}{ }^{4} \mathrm{P}_{11 / 2}^{\circ}-6_{21 / 2} \\
4 p^{2} \mathrm{P}_{1 / 2}^{\circ}-6 s^{2} \mathrm{~S}_{1 / 2} \\
4 p^{\prime}{ }^{4} \mathrm{~F}_{21 / 2}^{\circ}-14_{21 / 2}^{\circ}\end{array}$ \\
\hline $\begin{array}{l}4 \\
4 \\
2 \\
4\end{array}$ & $*$ & $\begin{array}{l}1511.718 \\
1511.694 \\
1510.363 \\
1510.045 \\
1508.645\end{array}$ & $\begin{array}{l}.700 \\
.362 \\
.045 \\
.644\end{array}$ & $\left\{\begin{array}{r}30 \\
40 \\
5 \\
40\end{array}\right.$ & $\begin{array}{r}8 \mathrm{R} \\
15 \mathrm{R} \\
8 \mathrm{R}\end{array}$ & $\begin{array}{r}15 \\
12 \\
150 \\
\\
70\end{array}$ & & $\begin{array}{l}66149.9 \\
66151.0 \\
66209.25 \\
66223.2 \\
66284.6\end{array}$ & $\begin{array}{l}4 p^{\prime}{ }^{4} \mathrm{~F}_{31 / 2}^{\circ}-12_{31 / 2}^{\circ} \\
4 s^{2}{ }^{2} \mathrm{D}_{11 / 2}-4 p^{\prime \prime 2} \mathrm{D}_{21 / 2}^{\circ} \\
4 p^{\prime}{ }^{4} \mathrm{~F}_{41 / 2}^{\circ}-12_{31 / 2} \\
4 p^{\prime}{ }^{4} \mathrm{P}_{21 / 2}^{\circ}-421 / 2\end{array}$ \\
\hline $\begin{array}{l}4 \\
4 \\
4 \mathrm{~h} \\
4 \mathrm{e}, \mathrm{i}^{*} \\
4 \mathrm{i}\end{array}$ & $\begin{array}{l}\mathrm{i}^{*} \\
*\end{array}$ & $\begin{array}{l}1504.034 \\
1503.653 \\
1503.109 \\
1499.077 \\
1499.052\end{array}$ & $\begin{array}{l}.044 \\
.649 \\
\\
.077 ? \\
.037\end{array}$ & $\begin{array}{r}5 \\
35 \\
30 \\
5\end{array}$ & $\begin{array}{l}5 R \\
5 R\end{array}$ & $\begin{array}{r}5 \\
25 \\
20 \\
20 \\
20\end{array}$ & & $\begin{array}{l}66487.9 \\
66504.7 \\
66528.8 \\
66707.7 \\
66708.8\end{array}$ & $\begin{array}{l}4 p^{\prime}{ }^{4} \mathrm{~F}_{31 / 2}^{\circ}-14_{21 / 2} \\
4 p^{\prime}{ }^{4} \mathrm{P}_{11 / 2}^{\circ}-8_{11 / 2} \\
{ }^{1} p^{\prime}{ }^{4} \mathrm{~F}_{31 / 2}^{\circ}-20_{21 / 2} ? \\
4 p^{\prime}{ }^{4} \mathrm{~F}_{31 / 2}^{\circ}-21_{41 / 2}^{\circ}\end{array}$ \\
\hline $\begin{array}{l}4 \\
1 \\
4 \\
2\end{array}$ & * & $\begin{array}{l}1497.410 \\
1496.001 \\
1493.133 \\
1492.121 \\
1488.926\end{array}$ & $\begin{array}{l}.410 \\
.043 \\
\times \\
.119 \\
.927\end{array}$ & $\begin{array}{r}40 \\
5 \\
40 \\
25 \\
10\end{array}$ & $\begin{array}{c}8 \mathrm{R} \\
1 \\
25 \mathrm{R} \\
\\
10 \mathrm{R}\end{array}$ & $\begin{array}{r}80 \\
\\
300 \\
30 \\
120\end{array}$ & & $\begin{array}{l}66782.0 \\
66844.9 \\
66973.27 \\
67018.7 \\
67162.50\end{array}$ & $\begin{array}{l}4 p^{\prime}{ }^{4} \mathrm{~F}_{41 / 2}^{\circ}-21_{41 / 2} \\
4 p^{\prime}{ }^{4} \mathrm{P}_{11 / 2}^{\circ}-9_{21 / 2} \\
4 s^{2}{ }^{2} \mathrm{D}_{11 / 2}-8 p^{2} \mathrm{P}_{1 / 2}^{\circ} \\
4 p^{\prime}{ }^{4} \mathrm{P}_{11 / 2}^{\circ}-11_{21 / 2}^{\circ} \\
4 s^{2}{ }^{2} \mathrm{D}_{11 / 2}-6 f^{2} \mathrm{~F}_{21 / 2}^{\circ}\end{array}$ \\
\hline $\begin{array}{l}1 \\
4 \\
1 \\
1 \\
4\end{array}$ & & $\begin{array}{l}1486.065 \\
1482.139 \\
1478.216 \\
1477.016 \\
1471.862\end{array}$ & $\begin{array}{l}\times \\
.140 \\
.216 \\
.015 \\
.857\end{array}$ & $\begin{array}{c}40 \\
30 \\
50 \\
60 \\
30\end{array}$ & $\begin{array}{c}40 \mathrm{R} \\
10 \mathrm{R} \\
30 \mathrm{R} \\
30 \mathrm{R} \\
8 \mathrm{R}\end{array}$ & $\begin{array}{r}700 \\
80 \\
300 \\
400 \\
50\end{array}$ & & $\begin{array}{l}67291.81 \\
67470.1 \\
67649.11 \\
67704.07 \\
67941.2\end{array}$ & $\begin{array}{l}4 s^{2}{ }^{2} \mathrm{D}_{21 / 2}-4 p^{\prime \prime}{ }^{2} \mathrm{~F}_{31 / 2}^{\circ} \\
4 p^{\prime}{ }^{4} \mathrm{P}_{21 / 2}^{\circ}-6_{21 / 2} \\
4 s^{2}{ }^{2} \mathrm{D}_{21 / 2}-4 p^{\prime \prime}{ }^{2} \mathrm{P}_{11 / 2}^{\circ} \\
4 s^{2}{ }^{2} \mathrm{D}_{11 / 2}-4 p^{\prime \prime}{ }^{2} \mathrm{~F}_{21 / 2}^{\circ} \\
4 p^{\prime}{ }^{4} \mathrm{P}_{21 / 2}^{\circ}-7_{31 / 2}\end{array}$ \\
\hline
\end{tabular}


TABLE 1. Zn II spectrum below $2570 \AA$ A-Continued

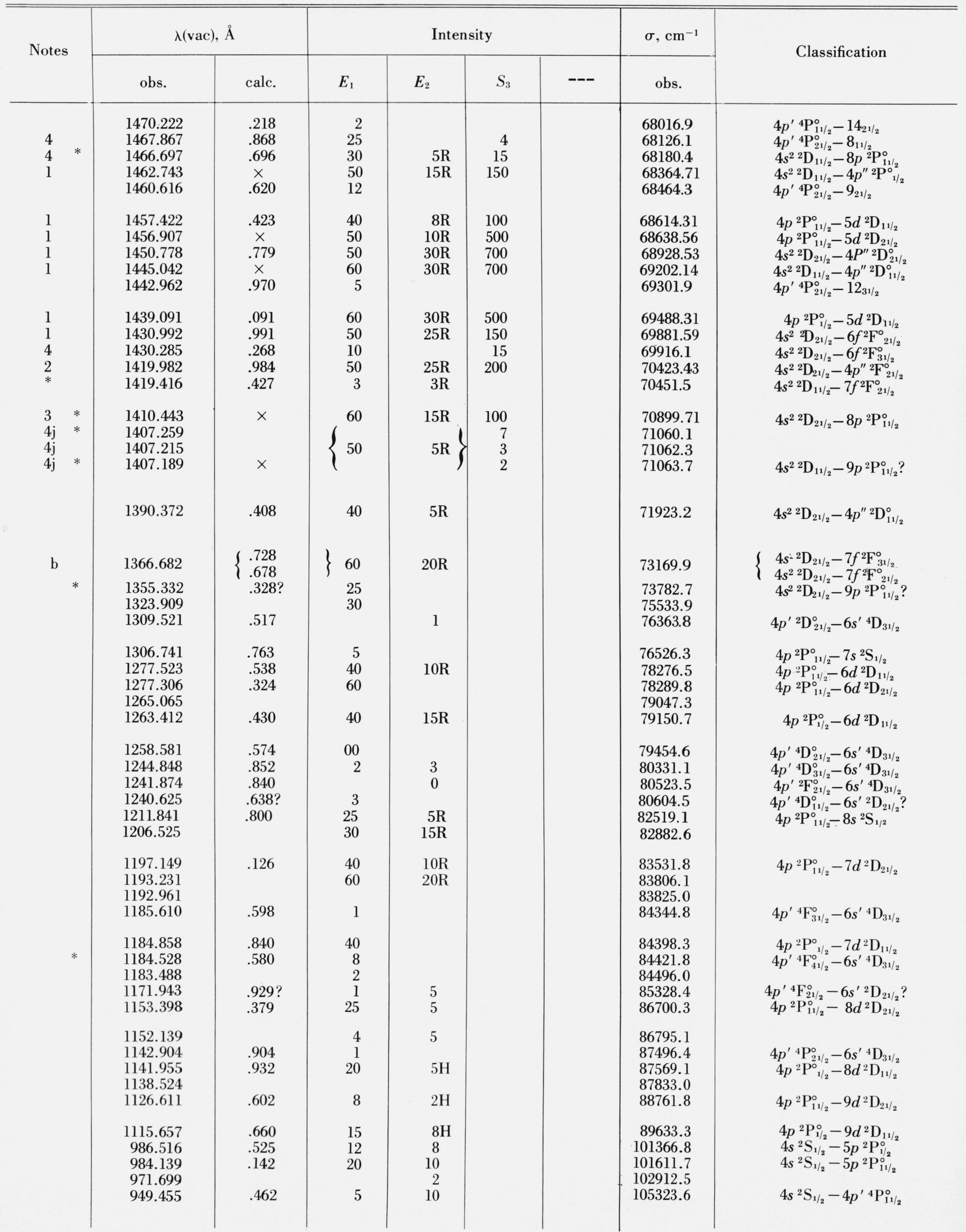


TABLE 1. Zn II spectrum below $2570 \not ̈$-Continued

\begin{tabular}{|c|c|c|c|c|c|c|c|}
\hline \multirow{2}{*}{ Notes } & \multicolumn{2}{|c|}{$\lambda(\mathrm{vac}), \AA$} & \multicolumn{3}{|c|}{ Intensity } & \multirow{2}{*}{$\begin{array}{c}\sigma, \mathrm{cm}^{-1} \\
\text { obs. }\end{array}$} & \multirow{2}{*}{ Classification } \\
\hline & obs. & calc. & $E_{1}$ & $E_{2}$ & --- & & \\
\hline \multirow{3}{*}{$\mathrm{f}$} & 938.719 & .713 & & & & 106528.2 & $4 s^{2} \mathrm{~S}_{1 / 2}-4 p^{\prime}{ }^{4} \mathrm{P}_{1 /}^{\circ}$ \\
\hline & 923.969 & .976 & & & & 108228.7 & $4 s^{2} \mathrm{~S}_{1 / 2}^{1 / 2}-4 p^{\prime}{ }^{4} \mathrm{~F}_{11 / 2}^{1 / 2}$ \\
\hline & 892.914 & .903 & 2 & 10 & & 111992.9 & $4 s^{2} \mathrm{~S}_{1 / 2}^{1 / 2}-4 p^{\prime}{ }^{4} \mathrm{D}_{11 / 2}^{\circ}$ \\
\hline \multirow[t]{6}{*}{$\mathrm{f}$} & 888.620 & .613 & & & & 112534.0 & $4 s^{2} \mathrm{~S}_{1 / 2}-4 p^{\prime}{ }^{4} \mathrm{D}_{1 / 2}^{\circ}$ \\
\hline & 881.060 & .064 & 1 & 15 & & 113499.6 & $4 s^{2} \mathrm{~S}_{1 / 2}-4 p^{\prime}{ }^{2} \mathrm{P}_{11 / 2}^{\circ}$ \\
\hline & 833.600 & .616 & & $1 \mathrm{H}$ & & 119961.6 & $4 s^{2} \mathrm{~S}_{1 / 2}-6 p^{2} \mathrm{P}_{11 / 2}^{\circ}$ \\
\hline & 779.163 & .159 & 6 & 10 & & 128342.9 & $4 s^{2} \mathrm{~S}_{1 / 2}-7 p^{2} \mathrm{P}_{11 / 2}^{1 / 2}$ \\
\hline & 778.112 & .098 & 2 & 5 & & 128516.2 & $4 s^{2} \mathrm{~S}_{1 / 2}-7 p^{2} \mathrm{P}_{1 / 2}^{\circ}$ \\
\hline & $\begin{array}{l}767.050 \\
755.223\end{array}$ & $\begin{array}{l}.038 \\
.202\end{array}$ & $\begin{array}{l}7 \\
4\end{array}$ & $\begin{array}{l}5 \\
5\end{array}$ & & $\begin{array}{l}130369.6 \\
132411.2\end{array}$ & $\begin{array}{l}4 s^{2} \mathrm{~S}_{1 / 2}-4 p^{\prime \prime}{ }^{2} \mathrm{P}_{11 / 2}^{\circ} \\
4 s^{2} \mathrm{~S}_{1 / 2}-8 p^{2} \mathrm{P}_{1 / 2}^{\circ}\end{array}$ \\
\hline * & $\begin{array}{l}747.358 \\
742.720\end{array}$ & $\begin{array}{l}.349 \\
.700\end{array}$ & $\begin{array}{l}3 \\
1\end{array}$ & $\begin{array}{l}5 \\
3\end{array}$ & & $\begin{array}{l}133804.7 \\
134640.2\end{array}$ & $\begin{array}{l}4 s^{2} \mathrm{~S}_{1 / 2}-4 p^{\prime \prime}{ }^{2} \mathrm{P}_{1 / 2}^{\circ} \\
4 s^{2} \mathrm{~S}_{1 / 2}-4 p^{\prime \prime}{ }^{2} \mathrm{D}_{11 / 2}^{\circ}\end{array}$ \\
\hline$*$ & 732.605 & $.572 ?$ & 00 & 1 & & 136499.2 & $4 s^{2} \mathrm{~S}_{1 / 2}-9 p{ }^{2} \mathrm{P}_{11 / 2}^{\circ} ?$ \\
\hline
\end{tabular}

${ }^{a} \mathrm{~A} \mathrm{ZnI}$ line also at this position belongs to a multiplet having negligible intensity in $E_{1}$. (In the region above $2400 \AA, E_{1}$ corresponds to $I_{4}$ in [9].)

${ }^{\mathrm{b}}$ Given in [1] or [9] as blend of $\mathrm{ZnII}$ and $\mathrm{Zn}$ III transitions. This explains an anomalously strong intensity in $E_{2}$ for three autoionizing $\mathrm{Zn}$ il lines.

${ }^{\mathrm{c}}$ Either a blend or the classification is incorrect. The indicated $\mathrm{Zn}$ II transition should not be observed in $E_{2}$.

III $\mathrm{Zn}$ III line now resolved from nearby $\mathrm{Zn}$ II line.

${ }^{d}$ Line belongs to either $\mathrm{Zn}$ II or $\mathrm{Zn}$ III. The close group at $1762.2 \AA$ has three resolved lines; our interpretation of measurements from three exposures $\left(S_{1}, S_{2}\right.$, and $\left.S_{3}\right)$ is that the group comprises four different lines. e Line assigned to $\mathrm{Zn}$ II only on the basis of a questionable classification.

Weak line from spark exposure $I_{3}$ [9]. (Intensities from this source are not listed here.) Wavelength from [1]; line is blended with a ghost on $S_{3}$ plate.

${ }^{\mathrm{h}} \mathrm{Line}$ is given as blend of $\mathrm{Zn}$ IV and $\mathrm{Zn}$ II in [1]; the new wavelength is entirely due to an unclassified $Z \mathrm{n}$ II transition.

The $E_{1}$ intensity appears to be too low.

'Interpretation of group not certain. There appear to be three lines, as given, with a possibility that the strongest $(1407.259 \AA)$ is either double or self-reversed. 
TABLE 2. Energy levels of $\mathrm{Zn} \mathrm{II}$


belong mainly to the $3 d^{9} 4 s 4 d$ configuration.

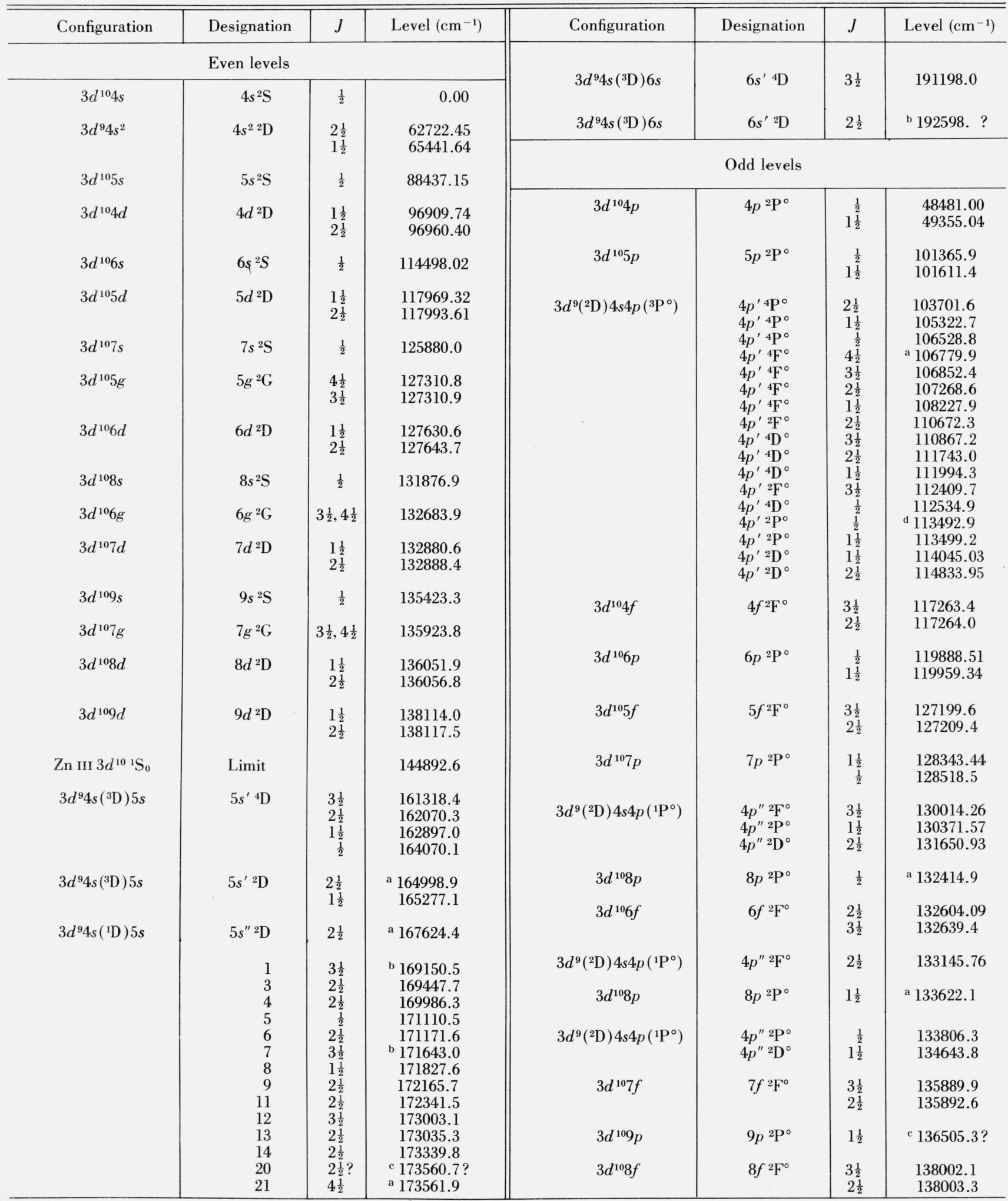

a New level from [4] or [5]. b J-value changed [5]. in [1], although an erroneous position occurred in the level table. 
from $5 s^{\prime}{ }^{4} \mathrm{D}_{31 / 2}$, and the new $4 p^{\prime}{ }^{4} \mathrm{~F}_{41 / 2}^{\circ}$ level $[4,5]$ was confirmed by the resolution and character of its strongest combination. ${ }^{5}$

Thirty of the classifications in table 1 are due to work reported in [4] and [5]. A few other new classifications are also listed.

Some previous ambiguities as to the spectrum assignments of certain lines are now resolved. None of the lines in table 1 has any appreciable intensity due to Zn IV in our sources. It appears from Dick's ionization stage assignments [9] that the experimental behavior of the weaker lines classified by Zn II levels was consistent with the classifications but, in itself, was often not conclusive. For the stronger lines a good separation of Zn II and Zn III was obtained [9]. Our sources allow a separation of $\mathrm{Zn}$ II and $\mathrm{Zn}$ I in the region above $1715 \AA$ for roughly this same group, and all the unclassified lines assigned to $\mathrm{Zn}$ II in this region are thus separated from both $\mathrm{Zn} \mathrm{III} \mathrm{and} \mathrm{Zn} \mathrm{I.}$ Below $1715 \AA$ there is no definite separation of weaker Zn II lines from possible Zn I lines.

Most of the unclassified lines in table 1 were observed by Crooker and Dick in one of their electrodeless discharges and assigned to $\mathrm{Zn}$ II on the basis of excitation behavior. Wavelengths for some additional unclassified lines that had $\mathrm{Zn}$ II character in a spark source may be found in [1]. Most of the lines previously classified by levels regarded as tentative (or now rejected) are not included in table 1 , since they were not assigned to $\mathrm{Zn}$ II on the basis of excitation characteristics alone. Conversely, the table

TABLE 3. Some additions to the $\mathrm{Zn}$ II line list of Crooker and Dick [1]

Unless otherwise noted, observations are from [9], with $E$ and $S$ corresponding to electrodeless-discharg e and spark intensities, respectively, as in [1]. Diffuse lines are denoted by "D".

\begin{tabular}{|c|c|c|c|c|c|}
\hline Note & $\lambda$ (air), $\AA$ & $E$ & $S$ & $\sigma, \mathrm{cm}^{-1}$ & Classification \\
\hline $\begin{array}{l}\mathrm{a} \\
\mathrm{b} \\
\mathrm{a} \\
\mathrm{a} \\
\mathrm{c} \\
\mathrm{b} \\
\mathrm{a} \\
\mathrm{b} \\
\mathrm{d} \\
\mathrm{e} \\
\mathrm{b} \\
\mathrm{b} \\
\mathrm{e} \\
\mathrm{b} \\
\mathrm{f} \\
\mathrm{f}\end{array}$ & $\begin{array}{l}6842.32 \\
6396.98 \\
5586.22 \\
5585.21 \\
5577.71 \\
3514.19 \\
3304.80 \\
3279.72 \\
3276.57 \\
3153.44 \\
3138.7 \\
2997.8 \\
2964.3 \\
2857.59 \\
2738.46 \\
2720.42\end{array}$ & $\begin{array}{c}1 \\
100 \\
(50) \\
15 \\
10 \\
20 \\
30 \mathrm{D} \\
30 \mathrm{D} \\
50 \\
10 \mathrm{D} \\
50 \mathrm{D} \\
10\end{array}$ & 5 & $\begin{array}{l}14610.9 \\
15628.1 \\
17896.2 \\
17899.5 \\
17923.5 \\
28448.0 \\
30250.3 \\
30481.7 \\
30511.0 \\
31702.2 \\
31851 . \\
33348 . \\
33725 . \\
34984.3 \\
36506.1 \\
36748.1\end{array}$ & $\begin{array}{l}5 d^{2} \mathrm{D}_{21 / 2}-6 f^{2} \mathrm{~F}_{21 / 2}^{\circ} \\
5 d^{2} \mathrm{D}_{21 / 2}-8 p^{2} \mathrm{P}^{\circ} \\
5 d^{2} \mathrm{D}_{21 / 2}-7 f^{2} \mathrm{~F}_{31 / 2}^{\circ} \\
5 d^{2} \mathrm{D}_{21 / 2}-7 f^{2} \mathrm{~F}_{21 / 2}^{\circ} \\
5 d^{2} \mathrm{D}_{11 / 2}-7 f^{2} \mathrm{~F}^{\circ}{ }^{21 / 2} \\
8 p^{2} \mathrm{P}_{11 / 2}^{\circ}-5 s^{\prime}{ }^{4} \mathrm{D}_{21 / 2}\end{array}$ \\
\hline
\end{tabular}

a Wavelength, classification from G. v. Salis, Ann. Physik [4] 76, 145 (1925).

${ }^{\mathrm{b}}$ Classification involves new level from [4] or [5]. Lines with questionable classifications may not belong to $\mathrm{Zn}$ II.

${ }^{\mathrm{c}}$ Wavelength from Shenstone and Gibson [3]. Intensity is a guess for unblended Zn II line, as actual line was blended with a strong $\mathrm{Zn}$ III line in [9].

dClassified by v. Salis.

e Upper level found by Crooker and Dick [1].

'Both wavelengths are from [3], for consistency. The $2738 \AA$ transition was listed in [1].

The line previously given for this transition, $1816.479 \AA$, [1] cannot arise from $5 s^{\prime}{ }^{4} \mathrm{D}_{31}$ Since this line is strong in $E_{1}$, does not appear in $E_{2}$, and is wide and weak (relative to $E_{1}$ ) in $S_{1}$, it arises from an (unknown) autoionization-broadened level. includes some new assignments to $\mathrm{Zn}$ II made on the basis of classifications by new levels.

Table 3 gives some classified lines above $2700 \AA$ that should be added to the list of Crooker and Dick [1] for completeness.

\section{Comments on Levels}

The $n f\left({ }^{2} \mathrm{~F}_{31 / 2}^{\circ}-{ }^{2} \mathrm{~F}_{21 / 2}^{\circ}\right)$ intervals obtained from the levels in table 2 are: ${ }^{6}$

$\begin{array}{ll}4 f & -0.6 \mathrm{~cm}^{-1} \\ 5 f & -9.8 \\ 6 f & +35.3 \\ 7 f & -2.7 \\ 8 f & -1.2\end{array}$

Although $f$ series with all or mostly negative intervals are frequently observed, this behavior has not been satisfactorily explained. It does seem clear that it is not generally due to interaction with known configurations. Such interactions can, in some cases, account for irregular variations of the intervals along the series. The $4 p^{\prime \prime}{ }^{2} \mathrm{~F}^{\circ}$ levels in $\mathrm{Zn}$ II are so positioned that interaction with them probably explains the relatively large positive interval of the $6 f^{2} \mathrm{~F}^{\circ}$ term ${ }^{7}$ and the increased negative interval of $5 f^{2} \mathrm{~F}^{\circ}$.

It seems best to rely entirely on the three-member $g$ series for the ionization energy. The Ritz formula yields a value of $144892.6 \mathrm{~cm}^{-1}$ for $\mathrm{Zn}$ III $3 d^{10}{ }^{1} \mathrm{~S}_{0}$ relative to the ground level of $\mathrm{Zn}$ II. An uncertainty of $\pm 2 \mathrm{~cm}^{-1}$ should take into account the effect of any errors in the levels.

All $\mathrm{Zn}$ II levels that appear to be well established are given in table 2. A few levels listed by Crooker and Dick [1] are omitted. Several of these were undesignated even levels (lying above the limit) given as questionable in [1]. (We have retained the originally assigned numbers as names for the remaining such levels.) The other deletions, and all except two of the new levels (both tentative), result from previously described work $[4,5]$.

\section{References}

[1] Crooker, A. M., and Dick, K. A., Can. J. Phys. 46, 1241 (1968). This paper includes an account of previous investigations of $\mathrm{Zn}$ II.

[2] Moore, C. E., Atomic Energy Levels, Nat. Bur. Stand. (U.S.), Circ. 467, Vol. II, 259 pages (1952). References to publications on $\mathrm{Zn}$ II earlier than [1] are given.

\footnotetext{
${ }^{6}$ Some oversights regarding the $7 f$ term in the level and line lists of [1] have been corrected. We also used data from references in table 3 to help determine some $n f$ levels.

"The inference that $6 f^{2} \mathrm{~F}^{\circ}$ has the most perturbed of these intervals is consistent with it position nearest to a perturbing $3 d^{9} 4 s 4 p$ level, $4 p^{\prime \prime}{ }^{2} \mathrm{~F}_{2 \psi_{2}}^{\circ}$. The observed $3 d^{9} 4 s^{2}{ }^{2} \mathrm{D}-3 d^{10} n f^{2} \mathrm{~F}$ multiplets (forbidden in the pure configuration approximation) probably arise mainly from the small interaction of the $3 d^{10} n f$ series with $3 d^{9} 4 s 4 p$. Thus, on our spectrograms, the $4 s^{2}{ }^{2} \mathrm{D}_{21 / 2}-6 f^{2} \mathrm{~F}_{21 / 2}^{\circ}$ transition at $1430.992 \AA$ is the strongest observed line belonging to any of these multiplets.
} 
[3] Shenstone, A. G., and Gibson, L. E., unpublished material. (The main results of this analysis were given in [2].)

[4] Martin, W. C., and Sugar, J., J. Opt. Soc. Am. 59, 1266 (1969). The present paper was given as the source of the experimental values for some levels and wavenumbers in Tables III, VI, and XII of this reference. These values were based on preliminary measurement of a zinc spark spectrogram. In some cases they differ by more than $1 \mathrm{~cm}^{-1}$ from the much more accurate values reported here.

[5] Martin, W. C., and Sugar, J., J. Res. Nat. Bur. Stand. (U.S.), 74A, (Phys. and Chem) No. 1, 7 (Jan.-Feb. 1970).

[6] Sugar, J., J. Opt. Soc. Am. 53, 831 (1963).
[7] Kaufman, V., and Andrew, K. L., J. Opt. Soc. Am. 52, 1223 (1962); Kaufman, V., and Ward, J. F., J. Opt. Soc. Am. 56, 1591 (1966); Kaufman, V., and Ward, J. F., Appl. Opt. 6, 43 (1967); Radziemski, L. J., Andrew, K. L., Kaufman, V., and Litzén, U., J. Opt. Soc. Am. 57, 336 (1967).

[8] Shenstone, A. G., Phil. Trans. Roy. Soc. (London) [A] 241, 297 (1948).

[9] Dick, K. A., The Spark Spectra of Zinc (Ph. D. Thesis, University of British Columbia, 1966).

(Paper 74A1-579) 